\title{
Vergangenheit - Gegenwart - Zukunft
}

\author{
Ursina Pally Hofmann
}

Dr. iur., Generalsekretärin der FMH

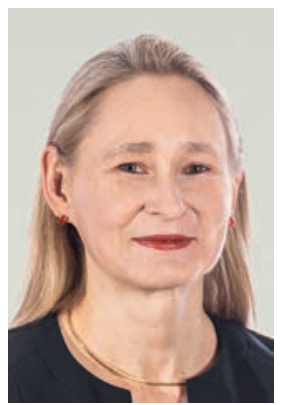

Traditionell werden im zwölften und letzten Monat die Jahresrückblicke angegangen. Sei es im privaten Umfeld, in der Presse oder anlässlich von Mitarbeitergesprächen; wir schauen zurück und prüfen, ob gut war, was war. Wir gehen meist davon aus, ein Jahr sei gut gewesen, wenn es das gebracht hat, was wir von ihm erwartet, und wir unsere Ziele erreicht haben. Nehmen wir unsere Prognose als Massstab, können wir im Rückblick wohl kaum je ein Jahr als "gut» bezeichnen. Denn wann immer wir zurückschauen, erkennen wir, dass vieles anders gekommen ist als geplant. Sollten wir deshalb unseren Jahresrückblick nicht auch danach beurteilen, ob es uns gelungen ist, Unerwartetes zu antizipieren und den Fokus anzupassen? Also eine Planänderung vorzunehmen und das, was auf den ersten Blick als unerwartete, allenfalls gar unerwünschte Veränderung daherkommt, als Chance zu packen? Was für ein Glück, dass nicht immer alles so kommt, wie wir es gerne hätten.

Zum Jahresende folgt auch immer ein Ausblick. Wir schauen nach vorne, planen für das nächste Jahr, hoffen auf bessere Zeiten oder befürchten eine Verschlechterung. Manchmal sehen wir diese bereits kommen. Doch die Zukunft bleibt ungewiss, bis sie zur

\section{Es ist bedauerlich, Chancen zu verpassen, weil die Bereitschaft fehlte, sie zu erkennen} und vom Plan abzuweichen.

Gegenwart wird, auch wenn wir regelmässig davon ausgehen, sie zumindest in groben Zügen schon heute zu kennen. Zukunft bedingt Offenheit, damit wir maximal von ihr profitieren können. Wie bedauerlich ist es doch, Chancen zu verpassen, weil die Bereitschaft fehlte, sie zu erkennen und vom Plan abzuweichen Risiken hingegen verwirklichen sich, auch wenn wir nicht hinschauen. Was hindert uns also daran, der Zukunft mit offenen Augen entgegenzutreten? Wir können zurückschauen und beurteilen, in die $\mathrm{Zu}$ kunft blicken und planen. Aber leben können wir nur in der Gegenwart. Wir erleben die Umwelt mit all unseren Sinnen, agieren und reagieren körperlich, emotional und rational. Nur die Gegenwart erlaubt es, Einfluss

Wir können zurückschauen und beurteilen oder in die Zukunft blicken und planen. Aber leben können wir nur in der Gegenwart.

zu nehmen und zu gestalten, auch für unsere Zukunft. Kein Moment kann nachgeholt werden - was von ihm bleibt, ist allenfalls die Erinnerung daran. Entscheidungen, die wir heute treffen, basieren auch auf den Erfahrungen, die wir in der Vergangenheit gemacht haben. Wie wir in der Gegenwart leben, hat also viel mit unserer Vergangenheit zu tun.

Vergangenheit, Gegenwart und Zukunft hängen zusammen. Die Zukunft von morgen gestalten wir heute basierend auf den Erfahrungen der Vergangenheit. Ein interessantes Spiel und eine faszinierende Zeitreise, die wir mit unserem Denken an die Vergangenheit, unserem Leben in der Gegenwart und dem Ausblick in die Zukunft treiben können. Ich versuche dreierlei: für meine Erinnerungen dankbar zu sein - auch wenn sie nicht nur schön sind; das Heute mit all meinen Möglichkeiten zu leben und die richtigen Pflöcke für die Zukunft einzuschlagen - auch wenn ich Fehler mache und ungewiss ist, welche Pflöcke die richtigen sind; und für die Zukunft offen zu sein - gerade weil ich nicht weiss, was sie mir bringen wird. 